\title{
Cloud Computing: Data Storage Protocols and Security Techniques
}

\author{
R. V. Dharmadhikari ${ }^{1 *}$, S. S. Turambekar $^{2}$, S. C. Dolli ${ }^{3}$, P K Akulwar ${ }^{4}$ \\ ${ }^{1}$ Computer Science and Engineering, Sanjay Ghodawat Institutes, Shivaji University, Atigre, India \\ ${ }^{2}$ Computer Science and Engineering, Sanjay Ghodawat Institutes, Shivaji University, Atigre, India \\ ${ }^{3}$ Computer Science and Engineering, Sanjay Ghodawat Institutes, Shivaji University, Atigre, India \\ ${ }^{4}$ Computer Science and Engineering, Sanjay Ghodawat Institutes, Shivaji University, Atigre, India \\ *Corresponding Author: rahuldharmadhikari5735@gmail.com
}

Available online at: www.isroset.org

Received: 10/Mar/2018, Revised: 26/Mar/2018, Accepted: 09/Apr/2018, Online: 30/Apr/ 2018

\begin{abstract}
Cloud computing has emerged as a new technology for hosting and delivering services over the internet. Cloud computing removes the necessity for users to plan ahead for resource provisioning. So it is appealing for business owners. Using cloud computing enterprises can start off with little resources as needed and later on increase the resources when needed. Cloud computing has made a huge impact in IT industry. However cloud computing is at its developing stages only. Still there are many issues to be considered. In this paper an overview of cloud computing is given. The concepts used in cloud computing is also highlighted. This paper also gives an idea about the data storage security and protocols which are used in cloud computing.
\end{abstract}

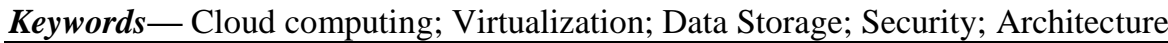

\section{INTRODUCTION}

Computing has become less of cost due to the development of processing and storage technologies and the success of internet. This kind of changing in technology and improvement in technologies has enabled the advent of a new computing model called the cloud computing. In cloud computing resources can be leased by the user on demand and these resources can also be released. This lease and release take place through internet. In cloud computing the conventional service providers are of two types. They are Infrastructure providers and service providers. Infrastructure providers manage the cloud platform and the resources for lease based on demand and the cost for the resources used. Service providers get the resources from infrastructure providers and give it to end users. Cloud computing has made a large impact on IT industry, where companies like Google, Amazon and Microsoft aim to provide powerful, reliable and cost efficient services.

\section{CLOUD COMPUTING}

Cloud computing can be defined as storing data in a collection of remote servers for storing and accessing data instead of personal computer or a server which is present locally. Here, users move their data to a remote server rather than storing data in a local server [1]. Cloud computing is similar to electricity grid, where users need not have a separate generator in their home for electricity. Instead they get electricity from the common electricity grid with less cost and it is also reliable. Similar way a user need not store their data in a local server. Instead they can store it in a cloud which is cheap as well as reliable and also users can access it anywhere from the world. Some features that make cloud computing attractive are:

- No payment in advance-Cloud uses a pay as you go model. i.e. it allows users to pay only for the resources they use.

- Less operational cost - Resources can be allocated and de allocated as per demand in a cloud. This is cost effective since resource can be released when there is less demand.

- Elasticity - The cloud infrastructure provides a large pool of data centres, so that cloud can handle any number of service demands.

- Available everywhere- The services in a cloud are web based. So users can access data from anywhere in the world. i.e. cloud is not restricted to location.

- Less maintenance cost- Since the infrastructure for a service is provided by cloud, the service provider need not worry about hardware operations. 
Cloud computing includes the following basic components

- Clients - Computers, laptops, PDA ${ }^{c e}$ s, mobile phones etc come under clients.

- Data Centre- This is a place where a collection of servers is kept.

- Distributed Server - These are the remote servers.

\section{A. Architectural model of cloud computing}

This section describes the architectural model of cloud. The cloud computing architecture contains

- Frontend - Thin client, thick client and mobile devices.

- Backend - storage, servers, routers, switches etc.

- Network - Internet.

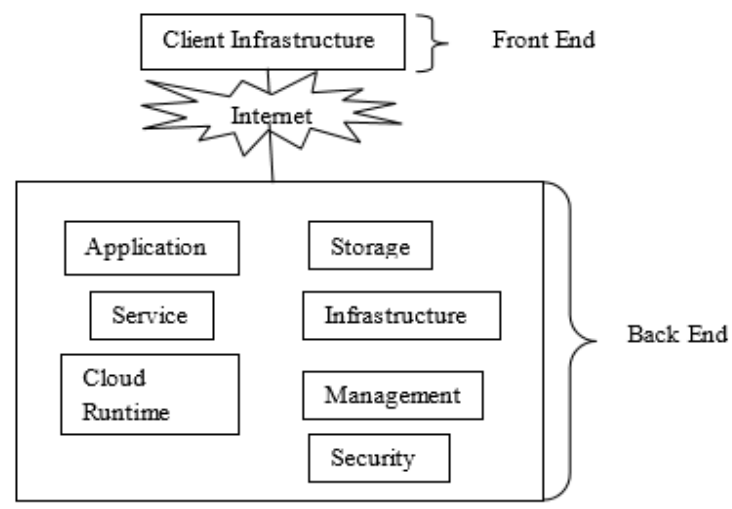

Figure1. Architectural Model of Cloud Computing.

The front end which comprises the client is connected to backend i.e. the cloud infrastructure through internet. [2]

\section{B. Business Model of Cloud Computing}

Cloud computing is a Service oriented business model. Here the hardware and software resources are based on demand. There are three services provided by cloud Infrastructure as a Service (IaaS), Platform as a service (PaaS) and Software as a Service (SaaS).

\section{1) $\operatorname{IaaS}$}

The idea behind this service is virtualization [5]. This layer provides hardware resources like network, storage, servers, routers etc which the clients can utilize through virtual machine. These services are provided by a service provider. These services are used to take care of various workloads [3]. Here the usage cost is calculated for how much resources are utilized per hour and companies pay the fees monthly or per year [1]. Some of the IaaS service providers are Amazon EC2, GoGrid etc.

\section{2) PaaS}

PaaS provides the resources to develop an application and services without buying the resources. It provides the software development framework. It provides a platform for software to be created, evaluated and implemented. Some of the PaaS providers are Google App Engine, Microsoft Azure etc.

\section{3) SaaS}

Here users can download the applications directly from the cloud using internet. In this model the users may have to pay for owing and using the application. The applications can also be accessed without downloading through web browser. SaaS provides application on demand over the internet. SaaS providers include SalesForce.com, Gmail etc.

\section{Cloud Deployment model}

This section describes the four types of cloud based on how it is implemented [5]

\section{1) Public Cloud}

When a cloud provider provides their service to public without any restriction then it is a public cloud. Public cloud has an important feature called multi tenancy i.e more than one user can access the cloud using internet at any time without any geographical limitation.

\section{a. Advantages \\ - No payment in advance for infrastructure.}

\section{b. Disadvantages}

- Public cloud cannot be trusted for Security.

\section{2) Private cloud}

This type of cloud model is owned and managed by a particular organization. This type of private cloud for a particular organization may be managed by the organization or by some other service provider. Here security is given by encryption and the resources are limited only to the organization.

\section{a. Advantages}

- Better in performance, Security and reliability.

\section{b. Disadvantages}

- It is similar to traditional server farms.

\section{3) Hybrid clouds}

This model of cloud comprises of both public and private cloud models. It is hybrid because the cloud is managed by a third party and used by an individual organization.

\section{a. Advantages}

- More flexibility 
- $\quad$ More Security

\section{b. Disadvantages}

- Designing a hybrid cloud is difficult.

\section{4) Virtual Private Cloud (VPC)}

VPC is a type of cloud created when a service provider creates a private clod by using public cloud resources. It overcomes the limitations of both public cloud and private cloud. The service provider can design their own polices in a VPN. The pubic cloud provider keeps aside some portion of the cloud for a private organization. The resources of this portion will not be shared with any other customers [6] VPC allows the data to be transferred securely between a public cloud and a private organization. This kind of security can be achieved using encryption, private IP addressing to user or allocating different VLAN to each user [7].

\section{E. Characteristics of cloud computing}

\section{1)Multi- tenancy}

Multi tenancy is an architecture in which multiple users are served by single instance of software. In a cloud the services provided by multiple service providers are located in a single area. Tenant is each customer. [8]. In this architecture each tenants can change the configuration according to their needs [9].

\section{2) Resource Pooling}

The pool of computing resources is dynamically assigned to each user. Because of dynamic resource assignment the service providers can manage the resources more effectively in terms of cost and usage. E.g. VM migration technology

\section{3) Geographically distributed}

The services provided by cloud are not restricted location wise. The services provided by cloud can be accessed in any part of the world through internet. Here the internet acts as a service delivery network.

\section{F. Service oriented}

Cloud is a service driven model. In cloud each service provider delivers their services in agreement to Service Level Agreement (SLA) agreed with their customers previously. SLA is necessary for each service provider.

\section{G. Resource provisioning}

Resource provisioning is an important feature of cloud computing. When a cloud provider accepts a request from a user it allocates the sufficient resources. There are three ways provisioning is done advanced provisioning, dynamic provisioning and user self provisioning. [10]

- Advance provisioning - The user reaches for the provider for services and the provider provides the resources in advance.

- Dynamic provisioning - The user is allocated more resources as needed and the resources are removed when not needed. Here the user pays according to how much they have used.

- User self provisioning- The user creates a user account through a web form and buys the resources from the cloud provider using credit card.

\section{H. Elasticity}

Cloud has the characteristics of scalability and flexibility to meet user demands. Adding and removing users, services, resources software etc is an easy task in cloud. [11]

\section{CONCEPT OF CLOUD COMPUTING}

The important concepts that are used in cloud computing is discussed below [12].

\section{A. Service Oriented Architecture}

Service oriented architecture is a group of services. These services are interoperable. The services can pass data between themselves or they can group for some other service.[14] The advantages of SOA in Cloud computing are [15]

- Cross platform communication - XML messages exchange is used to integrate multi platform like java, php etc. An XML file is a text file having meta- information tags.

- Loose coupling - Programs developed for SOA contains modules which are not dependant on each other.

- Reusability - Services can be reused in different system.

- $\quad$ Time saving - Because of service reusability time can be saved for construction and maintenance

- Availability - All the features are available for developers who need them.

\section{B. Virtualization}


The basis of cloud computing is Virtualization. Virtualization is creation of practical form but not in actual of something like operating systems, storage devices, servers or network resources [16]. Virtualization is one in three aspects

\section{1) Network Virtualization}

Combining the available network resources and splitting the resources and assigning to devices which is independent of each other. It reduces complexity of the network. It can be thought of dividing a hard disk into partitions which helps for easy managing the files.

\section{2) Storage Virtualization}

Storage Virtualization is pooling of storage from multiple storage devices and making them a single storage device. It is managed centrally.

\section{3) Server Virtualization}

Server Virtualization is abstracting the server resources from the server users. The user need not be concerned about the identity of server, operating system etc. The aim of server virtualization is to free the users from dealing with the complicated details of server.

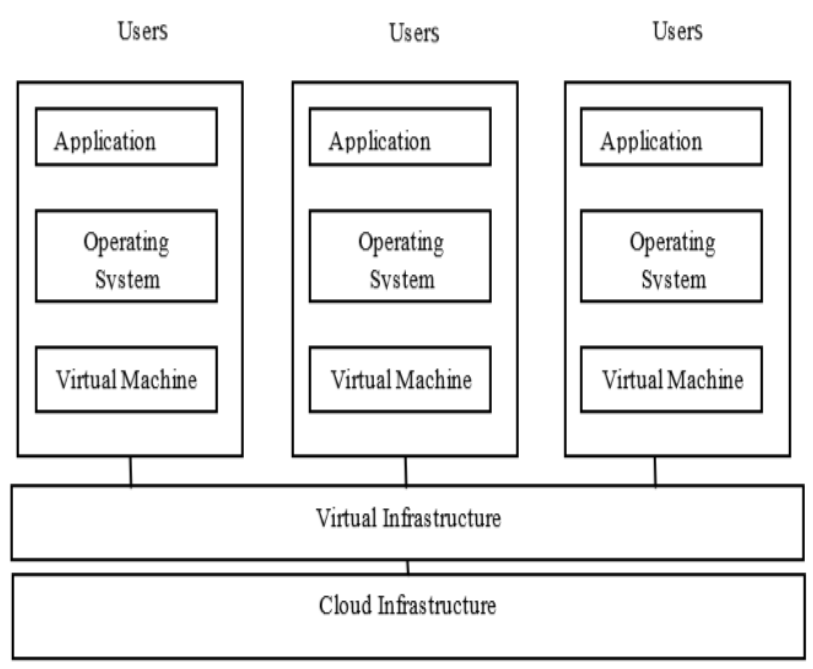

Figure 3. An overview of virtualization

\section{CLOUD PROVIDERS}

The company that provides services of cloud computing like IaaS, PaaS, and SaaS to other individuals or organization is called a cloud provider. Some of the famous cloud providers and their clouds are discussed below [18].

A. Amazon Ec2
EC2 is a large complex web service. Ec2 provides an API for creating instances with operating systems supported. EC2 can make computations through Amazon Machine Image (AMI). Some features of Amazon EC2 include S3, Cloud management, MapReduce cloud, Amazon Machine Image (AMI). Amazon Ec2 contains best tools for distribution, load balancing and cloud monitoring tools.

\section{B. Microsoft Azure}

Microsoft windows Azure is an on demand capacity builder in enterprise level. Users can avail the services on demand for price. Users have to use Azure API to use the services by Microsoft windows Azure. Some of the features of Microsoft Azure are worker role, web role, blob storage, table and drive storage.

\section{Google App Engine}

Google App Engine provides facilities for designing an application. It helps in development and deployment of applications in Java and Python. Google App Engine provides services with reliability, availability and scalability. Features include appspot and template. Good monitoring and management console.

\section{CLOUD STORAGE PROTOCOLS}

Some of the protocols used for data storage are discussed below [19]. 5.1. Simple Object Access Protocol (SOAP) SOAP uses transport protocols like HyperText Transfer Protocol (HTTP) and Simple Mail Transport Protocol (SMTP). SOAP defines standard rules for Extensible Markup Language (XML) based message exchange. Programs using SOAP can communicate with programs everywhere.

\section{A. Representational State Transfer (REST)}

REST is stateless software architecture. REST reads web pages containing XML. REST requires a provided server program to serve data and a provided client program to request data, so it is easier than SOAP.

\section{B. Fast and Secure Protocol (FASP)}

FASP is a bulk data moving technology. FASP is an alternative to Transmission Control Protocol based transport protocols. FASP resends packets only after the confirmation that the packet has been dropped.

\section{Common Internet File System (CIFS)}

Programs can make request to remote computers for files and services using CIFS through internet. CIFS is a variation of Server Message Block Protocol. CIFS uses TCP/IP protocol.

D. Network File System (NFS) 
Linux and UNIX operating system uses NFS. Using NFS users can view, store, and update files on a remote computer. It is a client server application. A system administrator can mount all or a portion of file system using NFS.

\section{E. Internet SCSI (ISCSI)}

It is an internet protocol. ISCSI helps transfer data over intranets and also manages long distance storage.

\section{DATA STORAGE SECURITY IN CLOUD COMPUTING}

Some of the existing techniques for data storage security in cloud computing are discussed below [20].

\section{A. Implicit storage security to data in online}

Here the data is partitioned in a way that each parts is secure implicitly and don't have the need to be protected by encryption. These parts are stored on various servers on the network and the location of these data is known only to the users. To modify the data the users need to know the location of the data and the server in which the data is located.

\section{B. Identify- based authentication}

Identify based Authentication is a hierarchical model with signature and encryption scheme. Here the client first sends the server a hello message with a random number $\mathrm{Cn}$ and session ID. The server responds with hello message with new random number $\mathrm{Sn}$.

\section{Public Auditing with Complete Data Dynamic Support}

Data integrity is very essential in severs. For providing dynamic data support, improvements are made in the existing scheme proof read of PDF or POR

\section{Efficient Third Party Auditing (TPA)}

For data storage security an algorithm called Bonch - Lynn Sachems is used. Using this algorithm the data blocks are signed before outsourcing the data into cloud. For error correction and data storage correction Reed Solomon Technique is used.

\section{E. Way of Dynamically Store Data in Cloud}

Data integrity is checked by an algorithm called Data Reading protocol algorithm and also data security is checked by the clients using effective automatic data reading algorithm. A mechanism called Flexible Distributed Storage Integrity Auditing Mechanism (FDSIAM) is used. This mechanism uses the homomorphism tokens, blocking erasure and unblocking factors and distributed erasure coded data.

\section{F. Effective and Secure Storage Protocol}

A secure storage protocol is implemented by using elliptic curve cryptography and sober sequence to ensure data integrity. Cloud customers execute data and software process protocol to ensure privacy of data and software before adding them to cloud. Challenge response protocol ensures that the data is not accessed by outsiders.

\section{G. Storage Security of Data}

To prevent attacks by intruder during transmitting data over internet a consistent and novel structure for providing security is introduced. It uses two-way hand shake based on token management by using homomorphism token with verification of erasure data. Using this scheme integration of storage correctness insurance and identification of misbehaving sever can be achieved.

\section{H. Secure and Dependable Storage Service}

A flexible distributed storage integrity auditing mechanism is introduced. This mechanism uses the homomorphism token and distributed coded data. This mechanism also supports block modification, deletion and append.

\section{Optimal Cloud Storage Systems}

The architecture contains three components. They are a Data Processor (DP), a Data Verifier (DV) and a Token Generator (TG). The Data processor processes the data before it is send to data verifier. The data verifier checks the data for any damage to the data. With the help of token generator cloud service providers can retrieve segments of data.

\section{J. File Storage and Security Management}

This system consists of a master server and a set of slave servers. The clients and slave servers are not connected by direct communication links. The clients request is processed by master server. The master server is also responsible for data backup. The files are stored in master server in the form of tokens. The files are chunked on slave server for data recovery.

\section{CONCLUSION}

Cloud computing is a technology that has recently emerged for managing and delivering services over the internet. Information Technology is rapidly changing by the rise of cloud computing. Computing has reached the next level. However even though cloud computing has its own benefits today's technologies are not up to the level to meet many of the requirements of cloud. Anyhow cloud computing has many advantages over the traditional computing. Due to virtualization cloud is able to achieve efficient resource utilization, elasticity and reducing cost. Still there are many issues that need to be addressed mainly in the area of security. This paper gives an overview of cloud computing, the deployment models of cloud and also the services offered by cloud. This paper also gives an idea about some of the concepts that are being used in cloud environment and also a brief overview of the security of the data stored in cloud and the protocols used in cloud. 


\section{REFERENCES}

[1] SurucheeV.Nandagaonkar, A.B Raut, "A Comprehensive Study on Cloud Computing" International Journal of Computer Science and Mobile Computing, vol, 3 Issue. 4, pg. 733-738, April 2014.

[2] http://cloudcomputingnet.com/cloud-computing-architecture/

[3] VikasGoyal, "Review: Layers architecture of cloud computing" International Journal of computing and Business research, Proceedings of ,I-Society $2012^{\text {ce at GKU }}$

[4] https://www.computenext.com/blog/when-to-use-saas-paas-andiaas/

[5] Qi Zhang, Lu Cheng, RaoufBoutaba, "Cloud computing: state-ofart and research challenges", Journal of Internet Services and Applications, pg. 7-18, April 2010

[6] https://www.techopedia.com/definition/26814/virtual-privatecloud-vpc.

[7] http://searchcloudapplications.techtarget.com/definition/virtualprivate-cloud-VPC.

[8] http://whatis.techtarget.com/definition/multi-tenancy

[9] Keiko Hashiume, David G Rosado, Eduardo Fernandez-medina, Eduardo B Fernandez, "An Analysis of Security Issues of cloud Computing", Journal of Internet Services and Applications, vol 4 Issue 5, 2013.

[10] http://searchcloudprovider.techtarget.com/definition/cloudprovisioning

[11] (http://erpbloggers.com/2013/07/the-five-essential-characteristicsof-cloud-computing/)

[12] Mladen A. Vouk, "Cloud Computing - Issues, Research and Implementation", Journal of Computing and Information Technology, pg 235-246, April 2008.

[13] (http://searchsoa.techtarget.com/definition/service-orientedarchitecture)

[14] (http://www.service-architecture.com/articles/web-services/service oriented_architecture_soa_definition.html)

[15] (http://searchservervirtualization.techtarget.com/definition/virtuali zation)

[16] T. Swathi, K. Srikanth, S. Raghunath Reddy," Virtualization in Cloud Computing", International Journal of Computer Science and Mobile Computing, vol, 3 Issue. 5, pg. 540-546, May 2014.

[17] B. Ramamurthy, K. Madurai," Concepts, Technologies and Business Implications "Wipro Chennai, 2010.

[18] (http://searchcloudstorage.techtarget.com/feature/Cloud-storagearchitecture-vs-local-storage)

[19] V. Spoorthy.M. Mamatha, B. Santhosh Kumar," A Survey on Data Storage and Security in Cloud Computing", International Journal of Computer Science and Mobile Computing, vol, 3 Issue. 6, pg. 306-313, June 2014 